\title{
Association between a color and a manual response activation modulates the response planning of typically developing children in a subsequent Simon task
}

\author{
Alexandre Coutté ${ }^{1}$ - Orianne Costini ${ }^{1,2} \cdot$ Laurent P. Ferrier $^{3} \cdot$ Evelyne Reymondet $^{1}$. $^{-}$ \\ Sylvane Faure ${ }^{1}$
}

Published online: 13 August 2015

(C) The Psychonomic Society, Inc. 2015

\begin{abstract}
The Simon effect usually refers to the observation that responding to a nonspatial feature of a stimulus is faster when the position of the stimulus (task-irrelevant) matches the position of the to-be-executed response. The Simon effect can disappear when the Simon task is preceded by a spatial compatibility task with an incompatible mapping. In this experiment, during a preliminary phase, 20 children had to decide whether the outline of a colored stimulus was dotted or continuous. Green stimuli were presented exclusively on the right, whereas red stimuli were presented on the left (the color was task-irrelevant). The participants then had to perform a Simon task. When the instructions required them to press either the left or the right button for red or green stimuli, respectively (Group A), the Simon effect on response latencies was not significant. With the opposite instructions (the right or left button for red or green stimuli, respectively; Group B), the Simon effect was significant on response latencies. The Simon effect was significant on movement times for both groups. These results suggest that during the preliminary phase, each color became associated with the manual response automatically activated by that color's position. In the subsequent Simon task, the presentation of the color activated the
\end{abstract}

Alexandre Coutté

coutte@unice.fr

1 Laboratoire d'Anthropologie et de Psychologie Cognitives et Sociales, Université de Nice-Sophia Antipolis, Nice, France

2 Université d'Angers, LUNAM, Angers, France

3 Laboratoire Epsylon, Université de Montpellier III, Montpellier, France associated response. This modulated the planning of the to-beexecuted response.

Keywords Simon effect $\cdot$ Implicit learning $\cdot$ Motor planning/ control $\cdot$ Action/perception coupling

Over the past few decades, a growing number of studies have used Simon tasks to explore the mechanisms underlying our interactions with the environment (see Hommel, 2011). In classical Simon tasks (Simon \& Rudell, 1967), participants have to discriminate a nonspatial attribute of a stimulus (e.g., color), while ignoring its location. The Simon effect refers to the fact that the motor response is executed faster when the spatial characteristics of the stimulus and the response match than when they do not, even if stimulus position is irrelevant to the task (Hommel, 2011).

Although most studies on the Simon effect have focused on the performance of adult participants, some authors have shown that the Simon effect can also be observed in children age 7 or older (Davidson, Amso, Cruess, Anderson, \& Diamond, 2006; Iani, Stella, \& Rubichi, 2014). Most of these studies have used Simon tasks to assess the executive functioning of children, and the results were discussed mainly in terms of cognitive inhibition and attentional control (Bialystok, Craik, Klein, \& Viswanathan, 2004; Davidson et al., 2006; Iani et al., 2014; Mandich, Buckolz, \& Polatajko, 2002; Martin-Rhee \& Bialystok, 2008). However, as has been suggested in previous studies with adult participants (see Hommel, 2011; Proctor, 2011), the Simon task also provides an experimental opportunity to explore the dynamic relationships between perception and action. Insofar as childhood is a critical period during which cognitive development involves complex dynamic interactions between multiple emerging systems (see D’Souza \& Karmiloff-Smith, 2011), 
studying the relationships between perception and action is crucial to our understanding of the development of stable, adaptive action patterns (see Whitall et al., 2006). Furthermore, this question is of clinical interest, since some studies have suggested that children with a developmental coordination disorder can be impaired in both coordination and control of the perception-action coupling (Whitall et al., 2006).

The present study was aimed more specifically at using a Simon task to explore the extent to which the coupling between perception and action in typically developing children might vary dynamically as a function of previous training.

Theoretical accounts of the Simon effect have generally claimed that perception and action are linked through two processing routes (Hommel, 2011; Kornblum, 1994; Proctor, 2011). When a stimulus is presented, (1) a direct route automatically activates a motor response corresponding to the spatial dimensions of the stimulus, and (2) an indirect route activates the to-be-executed response through arbitrary codes (e.g., the task instructions). The rapidity of the response depends on the correspondence of the spatial properties of the perceived stimuli and the produced actions. During a Simon task, the Simon effect is determined by the contributions of facilitation processes (Valle-Inclán, 1996) and interference processes (Couth, Gowen, \& Poliakoff, 2014; see Umiltà, Rubichi, \& Nicoletti, 1999).

The Simon effect is thought to affect different dimensions of motor control (Buetti \& Kerzel, 2009; Coutté, Olivier, \& Faure, 2015). Indeed, manual motor control is known to rely on planning and online control processes (Jeannerod, 1999; Schmidt, 1975). Although movement planning is generally prominent before and during movement initiation, online control gradually increases during movement execution (Glover, 2004). The respective contributions of planning and online control are dynamically modulated as a function of the task constraints. During a typical Simon task, the Simon effect usually has an impact on manual-response selection and programming, during both the preparation and execution of the manual response. The Simon effect is observed on response initiation latencies, response execution durations, and the kinematic parameters of the movement (Buetti \& Kerzel, 2009; Hietanen \& Rämä, 1995). More specifically, the strengths of the Simon effect on these response parameters vary as a function of the timing of response selection and programming. When stress is put on movement initiation (by the task instructions), the Simon effect impacts response execution durations but not response latencies (Buetti \& Kerzel, 2009; Rubichi \& Pellicano, 2004). By contrast, in a go/no-go Simon task, in which the to-be-executed motor response is precued before stimulus presentation, response precueing reduces the Simon effect on the movement parameters while preserving the Simon effect on response latencies (Buetti \& Kerzel, 2009; Rubichi \& Pellicano, 2004).
Going beyond this general framework, several authors have looked more specifically at the effect of training on the Simon effect. The Simon effect is known to be robust, even after extensive training (Simon, Craft, \& Webster, 1973). However, Proctor and Lu (1999) demonstrated that the Simon effect could be modulated by means of task-specific practice. These authors asked participants to practice a spatial stimulus-response compatibility (SRC) task in which they had to respond to the stimulus with the spatially incompatible response button (incompatible mapping). After this practice, they had to perform a Simon task, and the Simon effect was eliminated or even reversed (see also Iani, Rubichi, Gherri, \& Nicoletti, 2009; Proctor, 2011; Vu, 2011). Such an effect can be observed after a practice phase of 72 trials (Tagliabue, Zorzi, Umiltà, \& Bassignani, 2000). Moreover, Tagliabue et al. showed that this transfer effect from a spatial compatibility task to a Simon task is long-lasting (it remains present when the Simon task is performed immediately after, a day after, or a week after the spatial compatibility task) and can be observed both in adults and in children between 5 and 8 years of age.

To explain the elimination (or reversal) of the Simon effect after practice with an incompatible mapping, Tagliabue et al. (2000) suggested that an SRC task with an incompatible spatial mapping creates a short-term association between the stimulus location and the incompatible response. This shortterm association is the consequence of (1) the implicit, procedural knowledge developed through repeated execution of noncorresponding responses, and (2) explicit learning and intentional reinstating of a noncorresponding mapping rule $(\mathrm{Vu}$, 2011). This association remains active and influences performance in the subsequent Simon task by interfering with the overlearned long-term associations (e.g., the stimulus location usually activates a spatially corresponding manual response in Simon tasks; Proctor, Yamaguchi, Dutt, \& Gonzalez, 2013; $\mathrm{Vu}, 2011)$.

The experiments above explored the extent to which previously learned associations between a stimulus location and an executed manual response influence a subsequent Simon effect. However, to our knowledge, none of them looked at whether the association between a stimulus dimension (its color) and the response implicitly activated by its usual location can have a similar impact, even if the response is not executed.

The presentation of a lateralized stimulus, irrespective of its color, shape, or other nonspatial dimensions, is known to activate a spatially corresponding manual response. When a participant performs a color discrimination task in which the colored stimulus is always presented in the same place, the color should gradually become associated with the spatially corresponding activated response. Therefore, any further presentation of that color should automatically activate that manual response, no matter where it is presented. In a subsequent 
Simon task, the Simon effect should thus be modulated by the previously learned association.

Furthermore, one would expect that practicing the Simon task would also gradually influence the spatial mappings between stimuli and responses. Although previous experiments have suggested that the influence of a prior SRC task with an incompatible spatial mapping on a subsequent Simon effect does not significantly decrease over the Simon task session (see Proctor \& Lu, 1999; Proctor, Yamaguchi, \& Vu, 2007), this question has not been tested with a paradigm in which the color has been associated with an automatically activated (but unexecuted) response. Therefore, our aim was to carry out an exploratory study assessing whether the impact of a previously learned association (between a color and an automatically activated response) on the Simon effect varies as the Simon task is being executed.

Finally, the effect of previously learned associations on the Simon effect should be specific to response selection and programming processes. For Simon tasks in which the participants are not forced to select their response before its initiation, the Simon effect is usually observed during both response preparation and execution (Buetti \& Kerzel, 2009). However, the motor selection and programming of a response are thought to be generally prominent before and during movement initiation (see Glover, 2004). So, relative to execution time, the latency of response initiation should be affected more by the previously learned associations.

Although the relationships between perception and action are thought to be crucial to our understanding of the normal and pathological development of cognition (see D'Souza \& Karmiloff-Smith, 2011; Whitall et al., 2006), investigations using Simon tasks in children have been very few. Therefore, we specifically tested our hypothesis in children.

\section{Method}

\section{Participants}

Twenty right-handed children from 7 to 12 years of age $(M=$ 9.3, $S D=1.2$ ) participated in the study. Informed consent was obtained from the children and their parents. They were not aware of the purpose of the experiment. All of the children had normal or corrected-to-normal vision, with no learning disabilities or psychiatric history. Standardized tests were administrated to assess the participants' cognitive abilities. The Arrows, Comprehension, and Knock Tap subtests of the NEPSY (Korkman, Kirk, \& Kemp, 2003) assessed visuospatial processing, spatial command comprehension, and motor inhibition, respectively. The Stroop test (Albaret \& Migliore, 1999) assessed verbal inhibition. The Sky Search subtest of the Test d'evaluation de l'attention chez l'enfant (TEA-Ch; Manly, Robertson, Anderson, \& Mimmo-Smith, 2004) assessed visual selective attention. The Spatial Memory subtest of the Kaufman Assessment Battery for Children (KABC; Kaufman \& Kaufman, 2008) assessed spatial working memory. All of the children exhibited normal performance on all tests. Two groups were set up, as detailed below. No significant age difference was found between the groups $(F<1$, n.s.).

\section{Material}

Participants were seated in front of an 11.6-in. screen at a viewing distance of $60 \mathrm{~cm}$. The screen was positioned so that the stimuli appeared at eye level. The experimental procedure was controlled by the E-Prime software (Psychology Software Tools, Inc., Sharpsburg, PA).

The response device was set on a table between the participant and the screen. It had three buttons $(15 \mathrm{~mm} \times 15 \mathrm{~mm})$ : middle, left (Button 1), and right (Button 2). The left and right buttons were $5 \mathrm{~cm}$ from the middle button.

The visual display showed a red or green rectangle $(45 \mathrm{~mm}$ $\times 35 \mathrm{~mm}$ ) located in the area around (right, left, or upper) a central fixation cross (45-mm eccentricity).

\section{Procedure}

The experimental procedure consisted of two phases.

During Phase 1, colored rectangles were presented. After the display of the central cross to be fixated (for 1,000 ms), a rectangle, either green or red, was presented to the right or left of the cross. The participants were instructed to say aloud whether the outline of the rectangle was dotted or continuous (Fig. 1). The rectangle remained visible until the participant had responded. The response initiated a new trial.

During Phase 2, the participants had to perform a Simon task. At the beginning of each trial, they were asked to press the middle button with the index finger of the right hand while looking at the fixation cross. After 1,000 ms, a colored

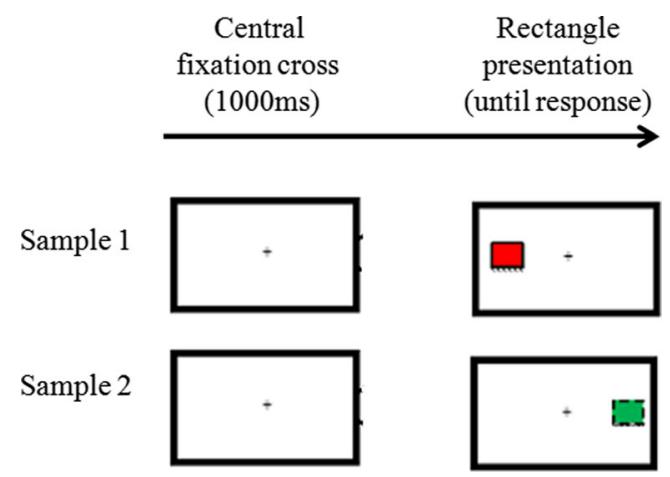

Fig. 1 Time courses of two sample trials for Phase 1. After display of the to-be-fixated central cross for 1,000 ms, a red (Sample 1) or green (Sample 2) rectangle was presented to the left (Sample 1) or the right (Sample 2), respectively 
rectangle (red or green) was presented to the right, to the left, or above the fixation point. Participants were instructed to release the middle button to execute the manual response as soon as possible. For ten participants (Group A), the instructions were congruent with the association implicitly formed during Phase 1; that is, in Phase 2, the participants were required to press either Button 1 (located on the left) or Button 2 (located on the right) when the rectangle was red or green, respectively. For the other ten participants (Group B), the instructions were not congruent with the association implicitly formed during Phase 1; that is, in Phase 2, participants were required to press Button 1 or 2 when the rectangle was green or red, respectively.

For each response, the computer recorded the reaction time in milliseconds (RT) - that is, the time between display onset and release of the middle button - and the movement time (MT) - that is, the time between release of the middle button and the pressing of one of the other two buttons. The visual display remained visible until the participant had pressed the response button.

\section{Experimental design}

For both groups, the Phase 1 block consisted of 80 randomly ordered trials that resulted from all possible combinations of the rectangle's color (right-located green vs. left-located red) and outline (dotted vs. continuous). The Phase 2 block consisted of 96 randomly ordered trials that resulted from all possible combinations of the rectangle's color (green vs. red) and location (right vs. left vs. upper; see Fig. 2).

\section{Results}

All participants made fewer than $1 \%$ wrong responses on Phase 1 . We ran the following analysis for the data collected on Phase 2. Wrong-response trials (1.4\% of the data) and trials on which the RTs were faster than $200 \mathrm{~ms}$ (anticipations) or slower than 2,000 ms (misses) $(9.8 \%$ of responses) were excluded from further analyses. In the remaining data, individual mean RTs and MTs were submitted to analyses of variance (ANOVAs) with (1) Trial Order (1st vs. 2nd half of trials) and (2) Correspondence (corresponding vs. noncorresponding vs. intermediate) as within-subjects factors, and Instructions (Group A vs. Group B) as a betweensubjects factor. The corresponding, noncorresponding, and intermediate conditions refer to trials in which, respectively, (1) the stimulus and the response corresponded spatially, (2) the stimulus and the response did not correspond spatially, and (3) the stimulus was presented above the fixation cross equidistantly from each of the response buttons. In order to test whether the numbers of trials were similar in each of the experimental conditions, after dividing the trials as a function of order, we ran the same ANOVA on the numbers of trials (see Table 1).

The ANOVA on the numbers of trials (see Table 1) revealed no significant effect of trial order, correspondence, $F(2,36)<1$, n.s., or instructions, $F(1,18)<1$, n.s. The trials tended to be slightly more numerous in the first half $(M=43.4$, $S D=4.8)$ than in the second half $(M=41.4, S D=6), F(1,18)$ $=3.4, p=.08$ : This resulted from more trials being discarded in the second half of the trials because the participants

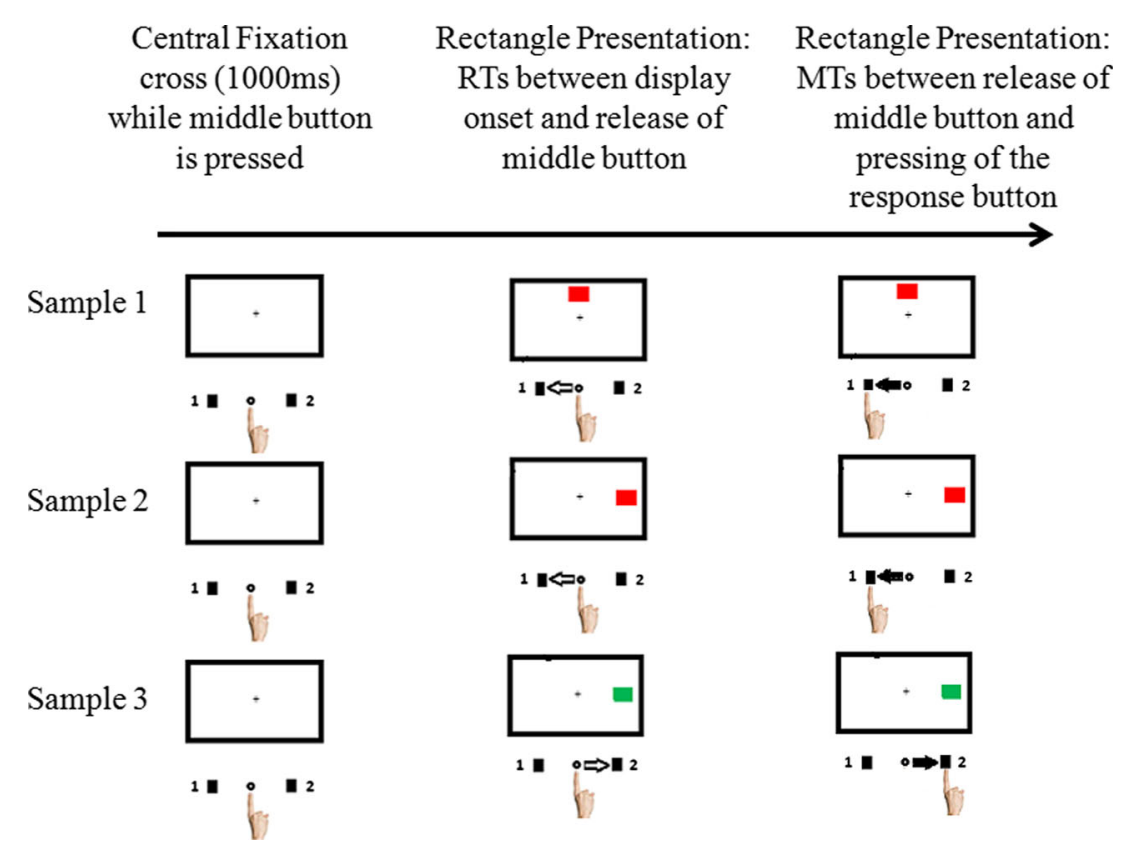

Fig. 2 Time courses of three sample trials for Phase 2 for a Group A participant. After display of the to-be-fixated central cross for 1,000 ms, a rectangle is presented. When the rectangle is red, the participant has to press Button 1 (e.g., Samples 1 and 2); when the rectangle is green, he or she has to press Button 2 (e.g., Sample 3) 
Table 1 Numbers of trials in each correspondence condition - that is, corresponding (C), noncorresponding (NC), and intermediate (I) - as a function of the instructions (Group A vs. Group B) and the trial order (1st vs. 2nd half of trials)

\begin{tabular}{llllr}
\hline & & C & I & NC \\
\hline Group A & 1st half of trials & $14.2(3,5)$ & $14.4(3,1)$ & $14.3(2,3)$ \\
& 2nd half of trials & $14.2(2,5)$ & $13.2(3,1)$ & $14(4,4)$ \\
Group B & 1st half of trials & $14.7(2,6)$ & $15.1(2,6)$ & $14(2,3)$ \\
& 2nd half of trials & $14.2(2,6)$ & $13.2(2,9)$ & $14.3(2,5)$
\end{tabular}

produced more wrong responses, misses, and anticipations. The two-way interactions between trial order and correspondence, $F(2,36)<1$, n.s., trial order and instructions, $F(1,18)<$ 1 , n.s., and correspondence and instructions, $F(2,36)<1$, n.s., were all nonsignificant. The interaction between trial order, correspondence, and instructions was also nonsignificant, $F(2,36)<1$, n.s.

The ANOVA on RTs (see Table 2) revealed no significant main effect of trial order, $F(1,18)<1$, n.s., correspondence, $F(2,36)=1.98, p=.15$, or instructions, $F(1,18)<1$, n.s. None of the two-way interactions were significant: trial order and correspondence, $F(2,36)<1$, n.s.; trial order and instructions, $F(1,18)<1$, n.s.; and correspondence and instructions, $F(2$, $36)=1.45, p=.25$. As expected, we did observe a significant interaction between trial order, correspondence, and instructions, $F(2,36)=3.31, p<.05\left(\eta^{2}=.16\right)$ on RTs (Fig. 3). We followed up this analysis by performing separate byinstructions analyses with Correspondence and Trial Order as within-subjects factors. For Group A, the statistical analysis revealed no significant effect of trial order, $F(1,9)<1$, n.s., or correspondence $F(2,18)<1$, n.s. Moreover, trial order did not interact significantly with correspondence, $F(2,18)=1.56, p$ $=.24$. For Group B, the statistical analysis revealed no significant effect of trial order, $F(1,9)<1$, n.s., and no significant interaction between trial order and correspondence, $F(2,18)=$ $1.86, p=.18$. However, we did observe a significant effect of correspondence, $F(2,18)=6.39, p<.01\left(\eta^{2}=.42\right)$ : Post-hoc Tukey tests revealed that RTs were significantly shorter $(p<$ $.05)$ in the corresponding condition $(M=479 \mathrm{~ms}, S D=109)$ than in the noncorresponding $(M=516 \mathrm{~ms}, S D=121)$ and intermediate $(M=522 \mathrm{~ms}, S D=138)$ conditions. The other comparisons failed to reach significance.

The ANOVA on MTs (see Table 2) revealed no significant main effects of trial order, $F(1,18)=1.4, p=.25$, or instructions, $F(1,18)=1.5, p=.24$. However, there was a significant correspondence effect, $F(2,36)=11.46, p<.001\left(\eta^{2}=.39\right)$ on MTs (Fig. 4). We followed up this analysis with post-hoc Tukey tests: MTs were significantly shorter in the corresponding condition $(M=393, S D=124)$ than in the intermediate $(M$ $=413 \mathrm{~ms}, S D=140 ; p<.05)$ and noncorresponding $(M=$ $451 \mathrm{~ms}, S D=123 ; p<.05)$ conditions. Other comparisons failed to reach significance. The interactions between trial order and correspondence, $F(2,36)<1$, n.s., trial order and instructions, $F(1,18)<1$, n.s., and correspondence and instructions, $F(2,36)<1$, n.s., were not significant, nor was the interaction between trial order, correspondence, and instructions, $F(2,36)=1.4, p=.27$.

\section{Discussion}

In the present experiment, we examined whether and to what extent the implicit learning of an association between a color and a spatial position may affect a subsequent Simon effect in children. Our statistical analysis brought out several results. First, the Simon effect on RTs was affected by the learning phase (Phase 1), in that the effect was significant only in Group B (i.e., when the instructions in Phase 2 were not congruent with the association implicitly formed during Phase 1). Second, the classic Simon effect was significant for MTs in both groups.

These results cannot be accounted for by cognitive or agerelated differences between the experimental groups. Respectively, all of the children exhibited normal performance on all standardized cognitive tests, and no significant age difference was found between the groups. Similarly, these effects cannot be interpreted as resulting from differences in the frequencies of corresponding, noncorresponding, and neutral trials in each half of the trials (see Stürmer, Leuthold, Soetens, Schröter, \& Sommer, 2002). Had that been the case, this

Table 2 Mean reaction times (RTs) and movement times (MTs), in milliseconds (with $S D \mathrm{~s}$ ), in each correspondence condition - that is, corresponding (C), noncorresponding (NC), and intermediate (I) - as a function of the instructions (Group A vs. Group B) and the trial order (1st vs. 2nd half of trials)

\begin{tabular}{|c|c|c|c|c|c|c|c|}
\hline & & \multicolumn{3}{|c|}{ 1st Half of Trials } & \multicolumn{3}{|c|}{ 2nd Half of Trials } \\
\hline & & $\mathrm{C}$ & $\mathrm{NC}$ & I & $\mathrm{C}$ & $\mathrm{NC}$ & I \\
\hline \multirow[t]{2}{*}{ RT } & Group A & $514(125)$ & $511(135)$ & $505(126)$ & 487 (123) & $524(146)$ & $488(104)$ \\
\hline & Group B & $462(74)$ & $522(105)$ & $518(114)$ & 496 (133) & 509 (135) & $525(159)$ \\
\hline \multirow[t]{2}{*}{ MT } & Group A & $426(178)$ & 488 (169) & $424(181)$ & 430 (187) & 500 (177) & $484(217)$ \\
\hline & Group B & $359(103)$ & $387(103)$ & $371(81)$ & $357(100)$ & $430(90)$ & 372 (127) \\
\hline
\end{tabular}



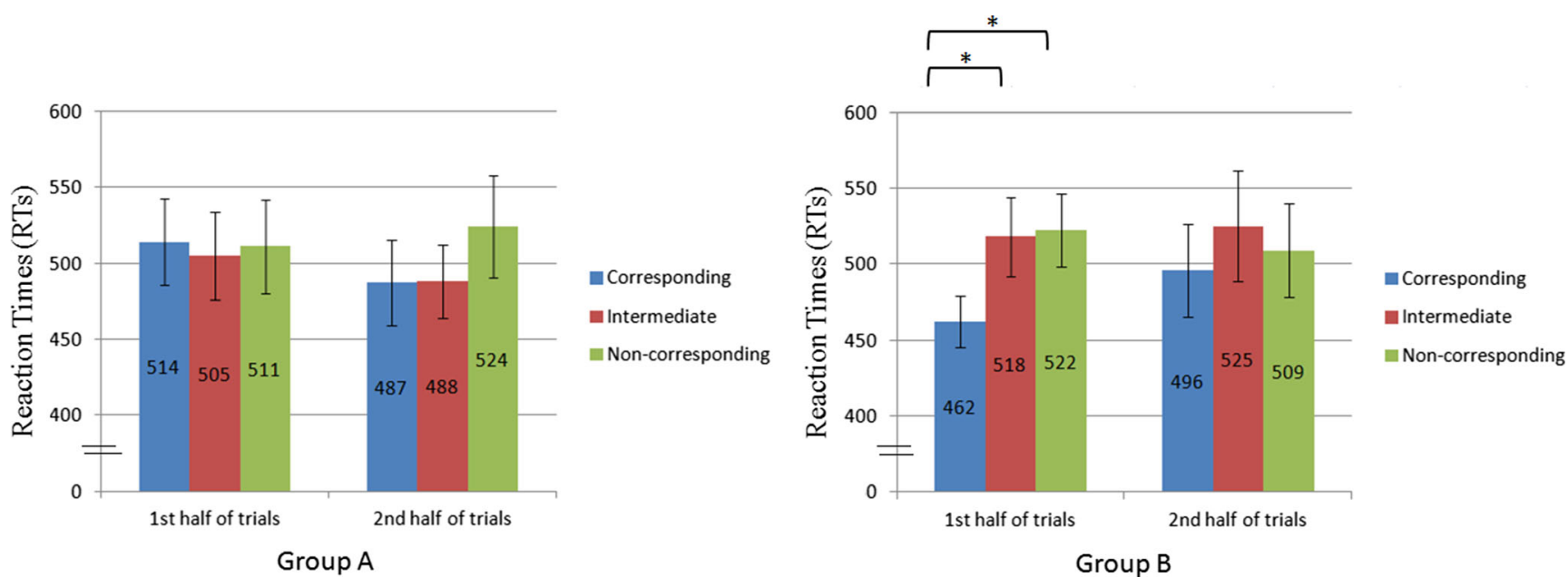

Fig. 3 Significant interaction between order, correspondence, and instructions on reaction times (RTs, in milliseconds) for Group A (left panel) and Group B (right panel). Significant comparisons $(p<.05)$ are labeled with asterisks. Error bars represent standard errors between participants

pattern of results would have been observed for both RTs and MTs. Moreover, the trials were randomly ordered, so the numbers of trials were similar in the different experimental conditions. The ANOVA on the number of trials confirmed this point.

In line with our expectations, the results pointed out the impact of previously learned associations between color and position on response planning during a subsequent Simon task. More specifically, during Phase 1, each stimulus color was always presented in the same location: Green and red rectangles were located on the right and left, respectively, for all the participants. Insofar as the presentation of a stimulus is known to activate a spatially corresponding manual response, each stimulus color was associated with an unexecuted manual response: a right manual response for green rectangles, and a left one for red rectangles.

During Phase 2, the previously learned short-term associations remained active and affected performance on the Simon task. More specifically, our results suggest that the presentation of a green rectangle during Phase 2 automatically

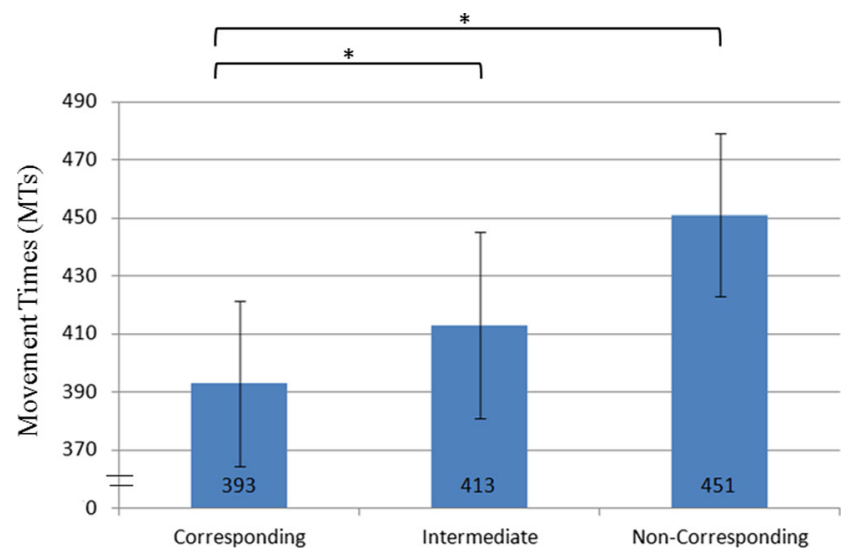

Fig. 4 Correspondence effect on movement times (MTs, in milliseconds). Significant comparisons $(p<.05)$ are indicated with asterisks. Error bars represent standard errors between participants activated selection of the right button for the manual response, whereas the presentation of a red rectangle automatically activated selection of the left button for the manual response. For the participants in Group A (instructions congruent with Phase 1), the responses activated by the colors were congruent with the experimental instructions. Therefore, when the rectangle was noncorrespondingly located (i.e., a left-located green rectangle or a right-located red rectangle), the rectangle's color activated a response that reduced the impact of the rectangle's location. The Simon effect, which is related to interference between the response activated by the stimulus location and the to-be-executed response, was therefore not significant for RTs. For the participants in Group B, the responses activated by the color were incongruent with the experimental instructions (i.e., they were asked to respond with the right or left button for a red or a green rectangle, respectively), and the interference brought about by the stimulus color was thus added to the classic Simon effect. The to-be-executed response was therefore initiated less rapidly in the noncorresponding condition, and the Simon effect was significant. Unexpectedly, these results differed from those of Tagliabue et al. (2000) on one point: In our study, we observed the disappearance of the Simon effect in Group A, whereas in Tagliabue's study the transfer effect from a spatial compatibility task to a Simon task resulted in a reversed Simon effect. Two factors may account for this difference. First, in our study, the manual responses were activated but not executed during Phase 1. The association between the color of the stimulus and the manual response was thus probably weaker than for studies in which the participants performed a spatial stimulus-response compatibility task. Consequently, the Simon effect was less impacted. Second, the transfer effect is thought to be stronger in children than in adults (Tagliabue et al., 2000). Insofar as the children tested in our study were older (their ages ranged from 7 to 12 years) than those tested by Tagliabue et al. (whose ages ranged from 
5 to 8 years), the transfer effect may have accordingly been weaker.

Interestingly, even though we observed an interaction between instructions, trial order, and correspondence, the Simon effect did not vary significantly in Group B as a function of the trial order. Because of the plasticity of the cognitive architecture, one could have expected that practicing the Simon task would induce dynamic spatial remapping, and therefore a lesser impact of the previously learned short-term associations at the end of the task. Further experiments (notably, with more trials in the experimental block) will thus be needed to more specifically assess the stability of this influence of the previously learned short-term associations.

These short-term associations seem to mainly influence the motor planning (and selection) of the to-be-executed response before its initiation. Indeed, the statistical analyses highlighted a classic Simon effect on MTs: The to-be-executed response was biased toward the stimulus location (Buetti \& Kerzel, 2009). In other words, the previously learned association between a color and a location did not significantly impact the Simon effect during movement execution. In the framework of Glover's motor control model (2004), this result suggests that the previous learning phase affects the Simon effect via the motor planning of the to-be-executed response, but not via its online control. However, insofar as response selection and programming are thought to occur both before and during response execution in accordance with the task constraints (see Buetti \& Kerzel, 2009), further experiments with other task constraints (using more sensitive measures, such as movement kinematic parameters) will be needed to explore the extents to which the previous learning phase impacts the Simon effects solely before, or also during, response execution (see Rubichi \& Pellicano, 2004).

In sum, our results have several implications. (1) They suggest that the presentation of a stimulus activates a specific response; the repetition of this stimulus presentation then enables the associations of all its dimensions (notably its color) with this implicitly activated (but not executed) response. (2) This association can have an impact on a subsequent Simon task; the color presented activates the associated response, and therefore modulates the Simon effect. In other words, a transfer effect can be observed without practicing a locationrelevant compatibility task with an incompatible spatial mapping. In the framework of the dual-route model (cf. Hommel, 2011), our results suggest that several dimensions of a single stimulus (e.g., its location and its color, when this color has been previously associated with a specific manual response) can activate motor responses that conjointly impact the execution of the required response. (3) The association between a color and a manual response activation influences the Simon effect via response selection and programming, not via the online motor control of the response.
More generally, this experimental paradigm seems to be a good one for investigating the developmental dynamics of relationships between perception and action. The present study highlighted a specific modulation of these relationships in children ranging in age from 7 to 12 years. With regard to previous studies in which the Simon effect was found to be affected by the age of the participants (Davidson et al., 2006; Iani et al., 2014), further longitudinal studies may allow us to investigate more precisely the evolution of this effect as a function of the age of the children. Furthermore, this paradigm could provide a means of gaining insight into atypical development of movement and gestures in children with developmental coordination disorders, for whom difficulties in action planning and control have been widely observed (Gabbard, 2009; Wilson, Ruddock, Smits-Engelsman, Polatajko, \& Blank, 2012).

\section{References}

Albaret, J. M., \& Migliore, L. (1999). Manuel du test de Stroop (8-15 ans). Paris, France: Editions du Centre de Psychologie Appliquée.

Bialystok, E., Craik, F. I. M., Klein, R., \& Viswanathan, M. (2004). Bilingualism, aging, and cognitive control: Evidence from the Simon task. Psychology and Aging, 19, 290-303. doi:10.1037/ 0882-7974.19.2.290

Buetti, S., \& Kerzel, D. (2009). Conflicts during response selection affect response programming: Reactions towards the source of stimulation. Journal of Experimental Psychology: Human Perception and Performance, 35, 816-834.

Couth, S., Gowen, E., \& Poliakoff, E. (2014). Dissociating affordance and spatial compatibility effects using a pantomimed reaching action. Experimental Brain Research, 232, 855-864. doi:10.1007/ s00221-013-3798-4

Coutté, A., Olivier, G., \& Faure, S. (2015). Influence of goal and laterality of a prepared hand response on a co-occurring visual search. Swiss Journal of Psychology, 74, 55-60.

D'Souza, D., \& Karmiloff-Smith, A. (2011). When modularization fails to occur: A developmental perspective. Cognitive Neuropsychology, 28, 276-287. doi:10.1080/02643294.2011.614939

Davidson, M. C., Amso, D., Cruess Anderson, L., \& Diamond, A. (2006). Development of cognitive control and executive functions from 4 to 13 years: Evidence from manipulations of memory, inhibition, and task switching. Neuropsychologia, 44, 2037-2078. doi: 10.1016/j.neuropsychologia.2006.02.006

Gabbard, C. (2009). A Developmental systems approach for the study of motor development. In L. T. Pelligrino (Ed.), Handbook of motor skills: Development, impairment, and therapy (pp. 170-185). New York: Nova Science.

Glover, S. (2004). Separate visual representations in the planning and control of action. Behavioral and Brain Sciences, 27, 3-24. disc. 24-78.

Hietanen, J. K., \& Rämä, P. (1995). Facilitation and interference occur at different stages of processing in the Simon paradigm. European Journal of Cognitive Psychology, 7, 183-199.

Hommel, B. (2011). The Simon effect as tool and heuristic. Acta Psychologica, 136, 189-202. doi:10.1016/j.actpsy.2010.04.011

Iani, C., Rubichi, S., Gherri, E., \& Nicoletti, R. (2009). Co-occurrence of sequential and practice effects in the Simon task: Evidence for two 
independent mechanisms affecting response selection. Memory \& Cognition, 37, 358-367. doi:10.3758/MC.37.3.358

Iani, C., Stella, G., \& Rubichi, S. (2014). Response inhibition and adaptations to response conflict in 6- to 8-year-old children: Evidence from the Simon effect. Attention, Perception, \& Psychophysics, 76, 1234-1241. doi:10.3758/s13414-014-0656-9

Jeannerod, M. (1999). Visuomotor channels: Their integration in goaldirected prehension. Human Movement Science, 18, 201-218.

Kaufman, A. S., \& Kaufman, N. L. (2008). KABC-II, batterie pour l'examen psychologique de l'enfant (2nd ed.). Paris, France: ECPA.

Korkman, M., Kirk, U., \& Kemp, S. (2003). NEPSY: Bilan neuropsychologique de l'enfant-Manuel. Paris, France: Les Editions du Centre de Psychologie Appliquée.

Kornblum, S. (1994). The way irrelevant dimensions are processed depends on what they overlap with: The case of Stroop and Simon-like stimuli. Psychological Research, 56, 130-135.

Mandich, A., Buckolz, E., \& Polatajko, H. (2002). On the ability of children with developmental coordination disorder (DCD) to inhibit response initiation: The Simon effect. Brain and Cognition, 50, $150-162$.

Manly, T., Robertson, I. H., Anderson, V., \& Mimmo-Smith, I. (2004). Test d'évaluation de l'attention chez l'enfant-TEA-Ch. Paris, France: Editions du Centre de Psychologie Appliquée.

Martin-Rhee, M. M., \& Bialystok, E. (2008). The development of two types of inhibitory control in monolingual and bilingual children. Bilingualism: Language and Cognition, 11, 81-93. doi:10.1017/ S1366728907003227

Proctor, R. W. (2011). Playing the Simon game: Use of the Simon task for investigating human information processing. Acta Psychologica, 136, 182-188.

Proctor, R. W., \& Lu, C.-H. (1999). Processing irrelevant location information: Practice and transfer effects in choice-reaction tasks. Memory \& Cognition, 27, 63-77. doi:10.3758/BF03201214

Proctor, R. W., Yamaguchi, M., Dutt, V., \& Gonzalez, C. (2013). Dissociation of S-R compatibility and Simon effects with mixed tasks and mappings. Journal of Experimental Psychology: Human Perception and Performance, 39, 593-609. doi:10.1037/a0029923

Proctor, R. W., Yamaguchi, M., \& Vu, K.-P. L. (2007). Transfer of noncorresponding spatial associations to the auditory Simon task. Journal of Experimental Psychology: Learning, Memory, and Cognition, 33, 245-253. doi:10.1037/0278-7393.33.1.245
Rubichi, S., \& Pellicano, A. (2004). Does the Simon effect affect movement execution? European Journal of Cognitive Psychology, 16, $825-840$.

Schmidt, R. A. (1975). A schema theory of discrete motor skill learning. Psychological Review, 82, 225-260. doi:10.1037/h0076770

Simon, J. R., Craft, J. L., \& Webster, J. B. (1973). Reactions toward the stimulus source: Analysis of correct responses and errors over a fiveday period. Journal of Experimental Psychology, 101, 175-178. doi: 10.1037/h0035766

Simon, J. R., \& Rudell, A. P. (1967). Auditory S-R compatibility: The effect of an irrelevant cue on information processing. Journal of Applied Psychology, 51, 300-304.

Stürmer, B., Leuthold, H., Soetens, E., Schröter, H., \& Sommer, W. (2002). Control over location based response activation in the Simon task: Behavioral and electrophysiological evidence. Journal of Experimental Psychology: Human Perception and Performance, 28, 1345-1363. doi:10.1037/0096-1523.28.6.1345

Tagliabue, M., Zorzi, M., Umiltà, C., \& Bassignani, F. (2000). The role of long-term-memory links and short-term-memory links in the Simon effect. Journal of Experimental Psychology: Human Perception and Performance, 26, 648-670. doi:10.1037/0096-1523.26.2.648

Umiltà, C. A., Rubichi, S., \& Nicoletti, R. (1999). Facilitation and interference components in the Simon effect. Archives Italiennes de Biologie, 137, 139-149.

Valle-Inclán, F. (1996). The locus of the interference in the Simon effect: An ERP study. Biological Psychology, 43, 147-162.

$\mathrm{Vu}$, K.-P. L. (2011). Unintentional and intentional learning of noncorresponding stimulus-response associations in the Simon task. Acta Psychologica, 136, 217-224. doi:10.1016/j.actpsy.2010. 05.005

Whitall, J., Getchell, N., McMenamin, S., Horn, C., Wilms-Floet, A., \& Clark, J. E. (2006). Perception-action coupling in children with and without DCD: Frequency locking between task-relevant auditory signals and motor responses in a dual-motor task. Child: Care, Health and Development, 32, 679-692.

Wilson, P. H., Ruddock, S., Smits-Engelsman, B., Polatajko, H., \& Blank, R. (2012). Understanding performance deficits in developmental coordination disorder: A meta-analysis of recent research. Developmental Medicine and Child Neurology, 55, 217-228. doi: 10.1111/j.1469-8749.2012.04436.x 